\title{
Modelling of high-density laser-material interaction using fast level set method
}

\author{
H Ki, P S Mohanty and J Mazumder \\ Center for Laser Aided Intelligent Manufacturing, Department of Mechanical Engineering \\ and Applied Mechanics, The University of Michigan, Ann Arbor, MI 48109-2125, USA
}

Received 14 August 2000

\begin{abstract}
A high-energy-density laser beam-material interaction process has been simulated considering a self-evolving liquid-vapour interface profile. A mathematical scheme called the level-set technique has been adopted to capture the transient liquid-vapour interface. Inherent to this technique are: the ability to simulate merger and splitting of the liquid-vapour interface and the simultaneous updating of the surface normal and the curvature. Unsteady heat transfer and fluid flow phenomena are modelled, considering the thermo-capillary effect and the recoil pressure. A kinetic Knudsen layer has been considered to simulate evaporation phenomena at the liquid-vapour interface. Also, the homogeneous boiling phenomenon near the critical point is implemented. Energy distribution inside the vapour cavity is computed considering multiple reflection phenomena. The effect of laser power on the material removal mode, liquid layer thickness, surface temperature and the evaporation speed are presented and discussed.
\end{abstract}

\section{Introduction}

Laser beams are currently being used for various manufacturing processes such as joining, precision machining and surface modification. Nevertheless, utilization of its full potential has a long way to go, primarily due to the lack of understanding of many associated process physics. When a laser beam irradiates a solid target, many complex phenomena take place, depending on the laser power density and the material characteristics. Figure 1 shows a schematic diagram of a high-energy-density laser beam-material interaction process. Upon absorption, the target becomes molten and subsequently vaporization occurs. The vapour flux generates a recoil pressure on the evaporating surface. Also, there exists a large temperature gradient at the liquid-vapour interface due to the spatial distribution of the laser beam energy, which generates a thermo-capillary force. The recoil pressure and the thermo-capillary force together provide the driving force for liquid ejection. Thus, the material is removed from the target both in vapour and liquid forms. Depending on the laser intensity, the liquid-vapour interface temperature may rise far beyond the normal boiling point. However, there exists a maximum temperature called the critical point that a liquid metal can attain. At the critical point the distinction between liquid and gas vanishes and only a fluid state exists [1-9]. Besides, the interface shape and the interaction physics are intimately coupled. In other words, the surface shape affects the interaction physics and vice versa.

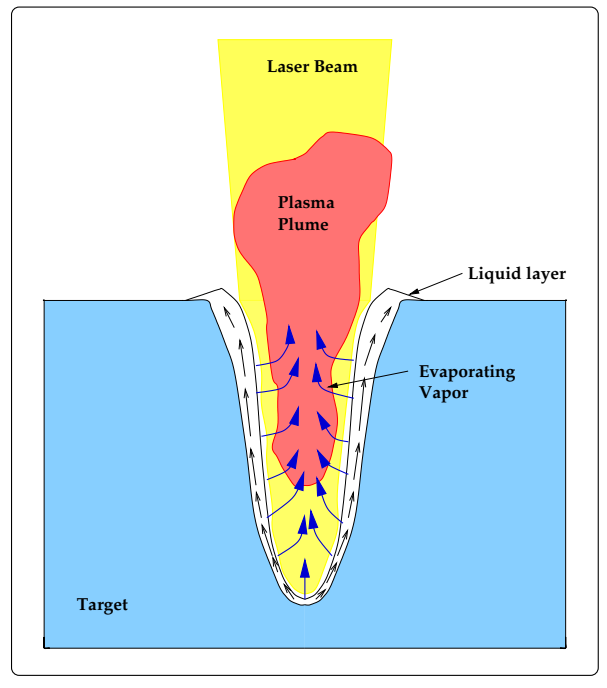

Figure 1. Schematic of laser-material interaction

Modelling of this process necessitates an interdisciplinary approach involving heat transfer, fluid mechanics, phase transformation physics as well as the tracking of the liquid-vapour interface.

There are many laser-material interaction models, but none of them has yet considered all the relevant process physics and the liquid-vapour interface evolution 

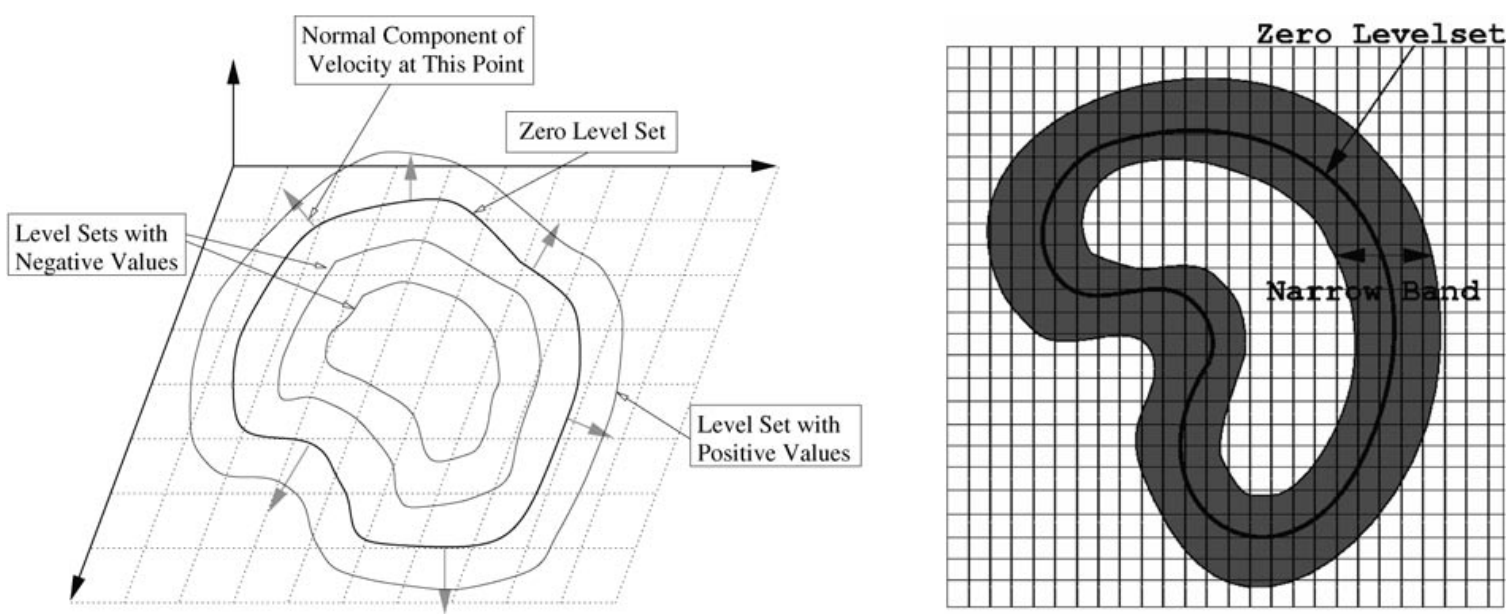

Figure 2. Definitions of the level-set functions.

self-consistently [10-12]. Chan and Mazumder [13] developed a one-dimensional steady-state laser-material interaction model considering the Knudsen layer and simple one-dimensional fluid flow. Kar et al [14] modelled the process considering multiple internal reflections, but the shape of the cavity was assumed a priori. Modest [15] developed a three-dimensional conduction model neglecting fluid flow and the evaporation physics. Ganesh et al $[16,17]$ recently developed a two-dimensional transient laser-material interaction model using the volume of fluid method for tracking the liquid-vapour interface. However, the evaporation physics was overly simplified. In most models $[4,13,16]$, a Clausius Clapeyron (CC) relation has been used to relate the pressure and temperature of the evaporating surface. However, due to the characteristic rapid heating by the laser beam, it is not appropriate. The liquid layer can have a huge amount of superheat and the pressure of the interface should be much lower than that estimated by the CC relation.

In the present study, a novel mathematical technique, called the level-set method, was implemented to capture the liquid-vapour interface self-consistently. Moreover, the emphasis was on the implementation of the evaporation physics and homogeneous boiling near the critical point. Fluid flow in the liquid layer was modelled by incorporating the thermo-capillary force and the recoil pressure. Multiple internal reflection phenomena are modelled considering a transient liquid-vapour interface. Since the material properties at near critical temperature are not available, many important properties have been extrapolated using the theoretically known material behaviour. Iron is used for the simulation purpose in this study. To the best of our knowledge this model is the first of its kind, where the interaction physics and the free surface motion have been coupled self-consistently.

\section{Mathematical model}

The computational techniques and the implementation details are presented in the following sections.

\subsection{Free surface evolution}

Melting and evaporation phenomena involve moving the liquid-vapour and solid-liquid boundaries. For the implementation of the process physics these boundaries must be tracked self-consistently. The efficient front tracking in two and three dimensions still remains a major challenge. Therefore, most of the laser beam-material interaction models either do not consider the influence of the interface geometry or assume the shape a priori, to simplify the computation. However, in recent years several elegant surface tracking mathematical schemes have evolved. Among them, the levelset method [18] is believed to be one of the best mathematical techniques and has been successfully used to capture complex free surface movement [19-24]. Most numerical techniques attempt to follow moving boundaries by putting a collection of marker points on the evolving front and then changing their positions to correspond to the moving front. This approach has many problems associated with it. Such schemes usually become unstable and blow up as the curvature builds around a cusp, since small errors in the position produce large errors in the determination of the curvature. In contrast, the level-set method is a technique that reformulates the equations of motion as a hyperbolic scalar conservation law with viscosity and some manageable boundary conditions. Then, these equations are solved with the techniques developed for gas dynamics. These techniques, based on high-order upwind formulations, are particularly attractive since they are highly stable, accurate and preserve monotonicity. The equation of motion for level set functions can be formulated as follows [24]:

$$
\frac{\partial \phi}{\partial t}+F_{l v}|\nabla \phi|+u_{a d v} \cdot \nabla \phi=0 .
$$

Here $\phi, \boldsymbol{u}_{a d v}$, and $F_{l v}$ are the level-set functions, fluid advection velocities and the normal velocities, respectively. In implementing this method, the interface we are interested in (liquid-vapour) is embedded as the zero level set (i.e. $\phi_{0}$ ) of one higher-dimensional space (figure 2). This zero level set is the surface where all the process physics should be implemented in order to evaluate the force that moves this surface. Except for the zero level set, i.e. the interface, the force functions are physically meaningless elsewhere in the computational 


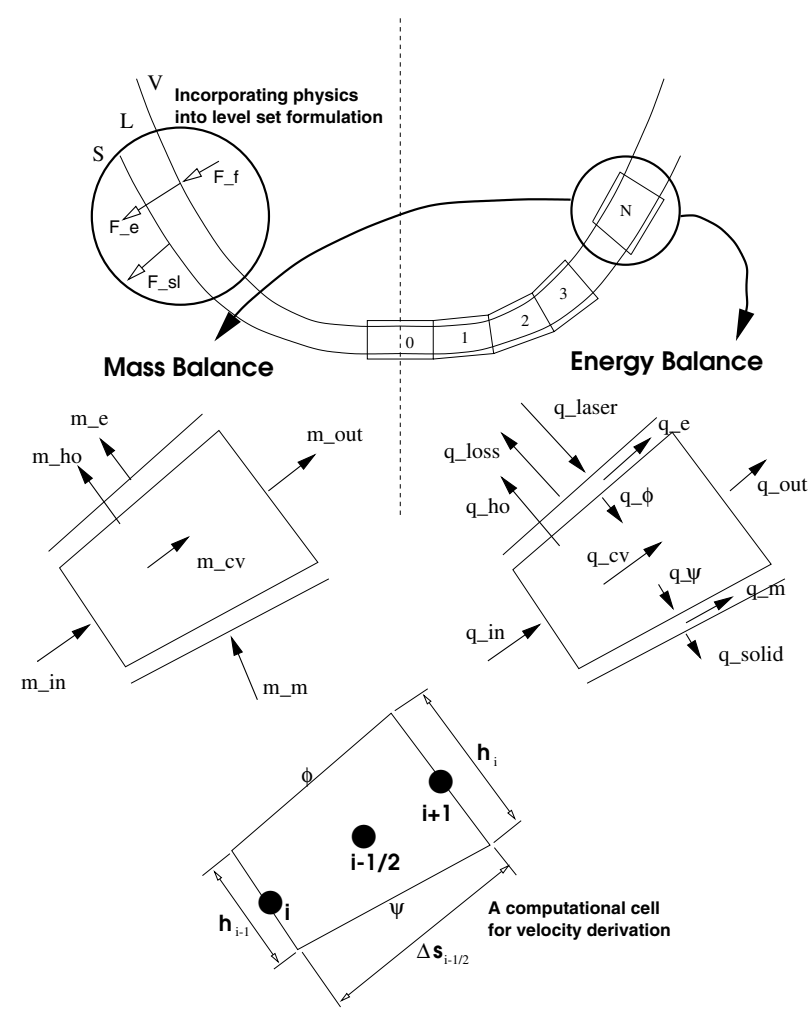

Figure 3. Mass and energy balance over a cell.

domain. The most challenging part of the whole computation is to extrapolate the force function to the whole space domain.

Figure 3 shows how the process physics are incorporated into the level-set formulation. This implicit representation allows one to track changes in topology and to calculate geometric quantities, such as the normal direction and the curvature, by capitalizing on the smoothness of the level-set function in a neighbourhood of the zero level set of interest. However, this embedding comes at a substantial price; one is now tracking all the level sets or the entire family of contours, not just the one that is of interest (i.e. $\phi_{0}$ ). The narrow band level set method, an advancement on this technique, solves this problem by focusing computational energy in a thin band around the front itself. Only the values of $\phi$ within the narrow band are updated. Values of $\phi$ at grid points on the boundary of the narrow band are frozen. When the front moves near the edge of the band, the calculation is stopped and a new band is built with the zero level-set interface at the centre. This rebuilding process is known as 're-initialization'. Using this approach, the operation count for the level-set method drops from $\mathrm{O}\left(N^{3}\right)$ in two dimensions to $\mathrm{O}\left(k N^{2}\right)$, where $N$ is the number of points in each space dimension and $k$ is the width of the narrow band [24]. The savings are substantial; they allow three-dimensional interface evolution problems to be handled with ease.

In high-energy-density applications, the melt layer is usually thin, and is determined from the energy and mass balance. This thin liquid layer forces the advection velocity to be perpendicular to the gradient of the level-set function, i.e. $\boldsymbol{u}_{a d v} \cdot \nabla \phi=0$. The speed function, $F_{l v}$ can be decomposed into two components and the final level-set equation can be written as

$$
\frac{\partial \phi}{\partial t}+\left(F_{e}+F_{f}\right)|\nabla \phi|=0
$$

where $F_{e}$ and $F_{f}$ are the speed functions due to evaporation and mass conservation respectively.

\subsection{Mass and energy balance}

Figure 3 presents a schematic diagram of the mass and energy balance in the liquid layer. Both convective heat and mass transfer are considered, keeping the model completely unsteady. The mass balance can be expressed as

$$
\dot{m}_{m}+\dot{m}_{\text {in }}=\dot{m}_{e}+\dot{m}_{\text {out }}+\dot{m}_{c v}+\dot{m}_{h o}
$$

where $\dot{m}_{\text {in }}$ and $\dot{m}_{\text {out }}$ are the mass flow rates due to fluid flow at the entrance and exit of the computational cell, respectively, and are obtained from the fluid flow equation. $\dot{m}_{h o}$ is the amount of mass loss due to homogeneous boiling, which is zero unless the liquid-vapour interface temperature is high enough; $\dot{m}_{m}$ is the mass flux into the liquid layer due to melting and can be expressed as

$$
\dot{m}_{m}=\rho_{s} A F_{s l}
$$

where $F_{s l}, \rho_{s}$ and $A$ are the melting speed, solid density and the bottom area of the cell, respectively; $\dot{m}_{e}$ is the evaporation mass flux at the liquid-vapour interface and is written as

$$
\dot{m}_{e}=\rho_{l} A F_{e}
$$

where $F_{e}$ is the contribution to the speed of the evaporating front only by evaporation and $\rho_{l}$ is the liquid density; and $m_{c v}$ is the mass accumulation rate in the cell, from which the time rate of liquid layer thickness can be obtained:

$$
\dot{m}_{c v}=\rho_{l} A \dot{d} .
$$

Here, $\dot{d}$ is the rate of increase in the liquid layer thickness. Unlike the mass balance, at the liquid-vapour and solid-liquid interfaces discontinuities exist in the energy flow due to latent heat. Stefan's condition is employed at the interfaces, and it can be written at the liquid-vapour interface as

$\dot{q}_{\text {laser }}=\dot{q}_{\text {loss }}+\dot{q}_{e}+\dot{q}_{\phi} \Longrightarrow A_{o} q_{e}=\dot{q}_{l o s s}+\rho_{l} L_{v} F_{e}-\left.k_{l} \frac{\partial T_{l}}{\partial n}\right|_{\phi}$

where $L_{v}, \partial / \partial n$ are the latent heat of vaporization and the directional derivative normal to the surface, respectively; and $\dot{q}_{\text {loss }}$ is the energy loss to the atmosphere due to the radiative and convective energy transfer. Stefan's condition at the solidliquid interface can be written as follows:

$$
\dot{q}_{\psi}=\dot{q}_{m}+\dot{q}_{\text {solid }} \Longrightarrow-\left.k_{l} \frac{\partial T_{l}}{\partial n}\right|_{\psi}=\rho_{s} L_{m} F_{s l}-\left.k_{s} \frac{\partial T_{s}}{\partial n}\right|_{\psi} .
$$

Here, $L_{m}$ is the latent heat of fusion, $\dot{q}_{\text {solid }}$ denotes the heat loss to the solid target, and is modelled using an analytical solution for a semi-infinite solid with a moving boundary [25].

The energy balance for a cell has the same form as the mass balance and is written as

$$
\dot{q}_{\phi}+\dot{q}_{i n}=\dot{q}_{\psi}+\dot{q}_{o u t}+\dot{q}_{c v}+\dot{q}_{h o}
$$




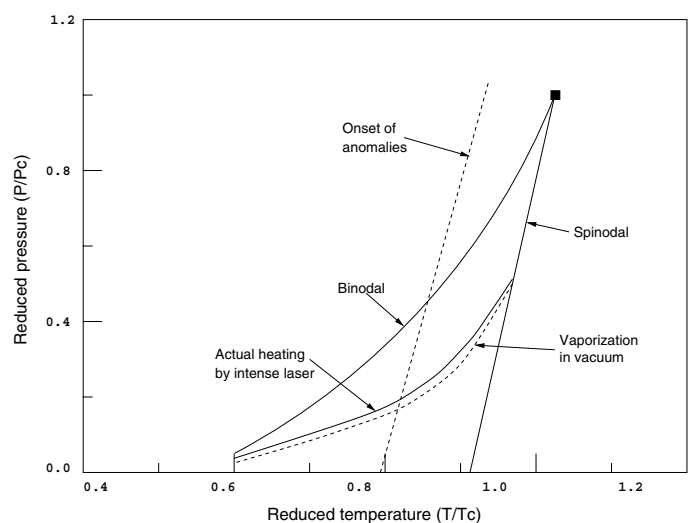

Figure 4. $P-T$ relationship (from [3]).

where $\dot{q}_{\text {in }}$ and $\dot{q}_{\text {out }}$ are the energy fluxes at the inlet and outlet of a cell, respectively, and $\dot{q}_{h o}$ denotes the heat loss due to homogeneous boiling; $\dot{q}_{\phi}$ and $\dot{q}_{\psi}$ are heat transmitted to the liquid layer through the liquid-vapour interface and heat flow to the solid-liquid interface from the liquid layer, respectively; and $\dot{q}_{c v}$ represents the heat accumulation rate in the cell and accounts for the temperature variation with time from the following relation:

$$
\dot{q}_{c v}=\rho_{l} A d C_{p} \frac{\mathrm{d} T_{m}}{\mathrm{~d} t} .
$$

Here, $\mathrm{d} T_{m} / \mathrm{d} t$ is the time rate of mean liquid temperature increase and $C_{p}$ is the specific heat. Since the temperature profile in the liquid layer is determined from four values such as the liquid-vapour and solid-liquid interface temperatures and temperature gradients at the liquid side of both interfaces, a cubic polynomial is assumed to interpolate the temperature profile.

\subsection{Evaporation at the liquid-vapour interface}

When the liquid-vapour interface temperature reaches the boiling point, evaporation begins to occur. It is known that there exists a very thin layer of several mean free paths, called the kinetic Knudsen layer, just outside the liquid-vapour interface. Across this layer the continuum hypothesis fails and steep changes in temperature, pressure and density occur. Therefore it is dealt with as a mathematical discontinuity at the interface. The jump conditions with back pressure were derived by Knight [26] and are omitted here.

The net mass loss due to evaporation can be calculated as [27]

$$
\dot{m}_{\text {evap }}=\rho_{s}\left(\frac{R T_{s}}{2 \pi}\right)^{1 / 2}-\rho_{v}\left(\frac{R T_{v}}{2 \pi}\right)^{1 / 2} \beta F^{-}(m)=\rho_{l} F_{e}
$$

where

$$
\begin{gathered}
F_{-}=\sqrt{\pi} m(-1+\operatorname{erf}(m))+\exp \left(-m^{2}\right) \\
G_{-}=\left(2 m^{2}+1\right)(1-\operatorname{erf}(m))-\frac{2}{\sqrt{\pi}} m \exp \left(-m^{2}\right) \\
\beta=\frac{2\left(2 m^{2}+1\right)\left(T_{v} / T_{s}\right)^{1 / 2}-2 \sqrt{\pi} m}{F_{-}+\left(T_{v} / T_{s}\right)^{1 / 2} G_{-}} .
\end{gathered}
$$

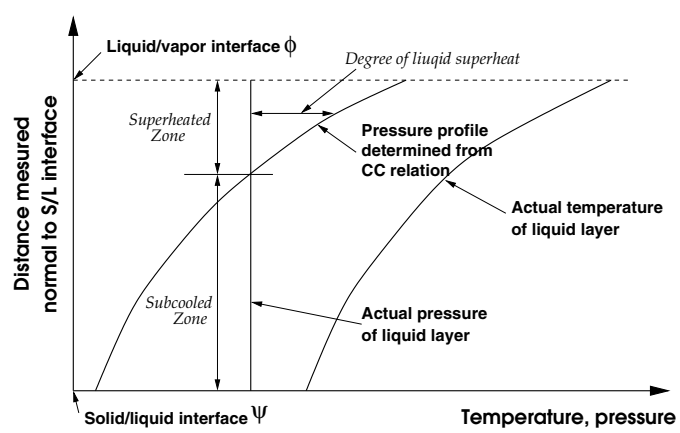

Figure 5. Degree of superheat in the liquid layer.

In the above expression, the factor $\beta$ is the modification factor which accounts the back-scattered flux [27] and $F_{e}$ is the speed function of the liquid-vapour interface due to evaporation. The degree of superheat is approximated using the momentum exchange balance across the Knudsen layer. Under thermodynamic equilibrium between a gas and liquid, the evaporating and back-scattered fluxes are equal and equally contribute to the surface pressure. Under vacuum, however, since there is no back-scattered flux, the interface pressure is around $50 \%$ of the saturation pressure. Rigorous calculation reveals that the pressure is around $0.56 P_{\text {sat }}\left(T_{S}\right)$ [9]. Thus the back-scattered flux is small compared to the evaporating flux, and the actual surface pressure can be expressed as

$$
P_{s} \cong \frac{\rho_{s} v_{s}+\rho_{v} v_{v}}{2 \rho_{s} v_{s}} P_{s a t}\left(T_{s}\right) .
$$

\subsection{Homogeneous boiling}

It has been reported that homogeneous boiling starts to occur directly beneath the liquid-vapour interface when the interface temperature reaches around $80 \%$ of the critical point due to a significant drop in surface tension and huge amount of liquid superheat (figure 4). The homogeneous boiling process acts as a heat sink, causing the liquid metal temperature to saturate.

Homogeneous nucleation phenomena are summarized in [28-30]. The homogeneous evaporation rate, which is number of bubbles with a critical radius per unit time per unit volume of liquid can be computed by

$$
J=N_{l}\left(\frac{6 \sigma}{\pi m\left(2-P_{l} / P_{v e}\right)}\right)^{1 / 2} \exp \left(\frac{-4 \pi r_{e}^{2} \sigma}{3 k_{B} T_{l}}\right)
$$

where $N_{l}, m, P_{v e}$ and $k_{B}$ are the number of liquid molecules per unit volume, the mass of one molecule, the pressure in the vapour bubble and the Boltzmann constant $(1.3807 \times$ $\left.10^{-23} \mathrm{~J} \mathrm{~K}^{-1}\right)$, respectively. The number of molecules in a bubble, $n_{e}$, is now

$$
n_{e}=\frac{V_{d} N_{A}}{v_{v} \bar{M}}
$$

where $V_{d}$ is the volume of a bubble and is expressed as

$$
V_{d}=\frac{4}{3} \pi r_{e}^{3}
$$

$\bar{M}$ is the molecular mass (55.85 for iron). Finally, the mass flux and energy flux due to homogeneous bubble generation near the critical point are

$$
\dot{m}_{h o}=J n_{e} m_{m}
$$


Table 1. Material properties for iron. ( $p_{c r}$ is obtained by substituting $T_{c r}$ to the CC relation. For simulation, the material properties are extrapolated to the critical point.)

\begin{tabular}{|c|c|c|}
\hline Property & Symbol & Value \\
\hline Melting temperature $(\mathrm{K})$ & $T_{m}$ & 1809.0 \\
\hline Normal boiling temperature $(\mathrm{K})$ & $T_{b}$ & 3133.0 \\
\hline Critical point temperature $[35](\mathrm{K})$ & $T_{c r}$ & 9250.0 \\
\hline Critical point pressure $(\mathrm{Pa})$ & $p_{c r}$ & $8.973 \times 10^{8}$ \\
\hline Liquid density $\left(\mathrm{kg} \mathrm{m}^{-3}\right)$ & $\rho_{l}$ & 6518.5 \\
\hline Solid density $\left(\mathrm{kg} \mathrm{m}^{-3}\right)$ & $\rho_{s}$ & 7870.0 \\
\hline Kinematic viscosity $\left(\mathrm{m}^{2} \mathrm{~s}^{-1}\right)$ & $v_{l}$ & $4.936 \times 10^{-7}$ \\
\hline Surface tension & $\sigma$ & See section 2.5 \\
\hline Latent heat of vaporization $\left(\mathrm{J} \mathrm{kg}^{-1}\right)$ & $L_{v}$ & $6.3639 \times 10^{6}$ \\
\hline Latent heat of fusion $\left(\mathrm{J} \mathrm{kg}^{-1}\right)$ & $L_{m}$ & $2.7196 \times 10^{5}$ \\
\hline Solid thermal conductivity $\left(\mathrm{W}(\mathrm{m} \mathrm{K})^{-1}\right)$ & $k_{s}$ & 40.96 \\
\hline Liquid thermal conductivity $\left(\mathrm{W}(\mathrm{m} \mathrm{K})^{-1}\right)$ & $k_{l}$ & 43.99 \\
\hline Liquid constant-pressure specific heat $\left(\mathrm{J}(\mathrm{kg} \mathrm{K})^{-1}\right)$ & $C_{p l}$ & 804.03 \\
\hline Solid constant-pressure specific heat $\left(\mathrm{J}(\mathrm{kg} \mathrm{K})^{-1}\right)$ & $C_{p s}$ & 658.63 \\
\hline Liquid thermal diffusivity $\left(\mathrm{m}^{2} \mathrm{~s}-1\right)$ & $\alpha_{l}$ & $8.39 \times 10^{-6}$ \\
\hline Solid thermal diffusivity $\left(\mathrm{m}^{2} \mathrm{~s}-1\right)$ & $\alpha_{s}$ & $7.90 \times 10^{-6}$ \\
\hline Laser absorptivity for flat surface & $A_{o}$ & 0.15 \\
\hline Liquid enthalpy [35] & $h_{l}$ & $\left(-0.18626+8.2516 \times 10^{-4} T\right) \times 10^{6}$ \\
\hline
\end{tabular}

$\mathrm{I}=10^{7} \mathrm{~W} / \mathrm{cm}^{2}$

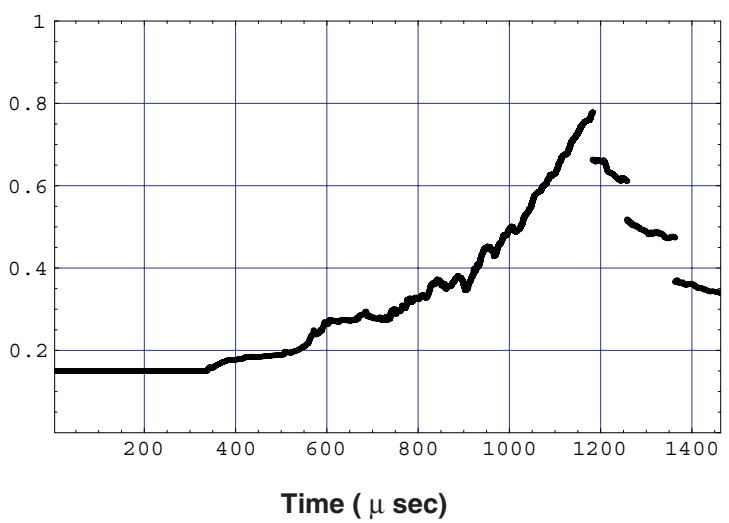

$\mathrm{I}=10^{8} \mathrm{~W} / \mathrm{cm}^{2}$

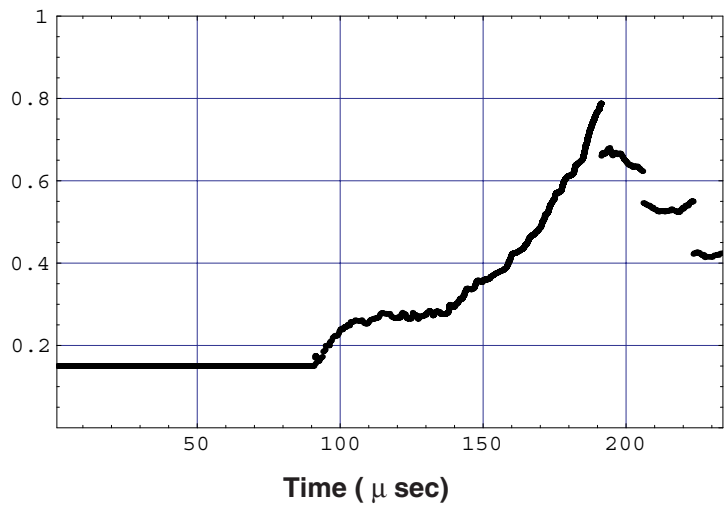

Figure 6. Effective laser absorptivity of a laser-created cavity.

and

$$
\dot{q}_{h o} \cong J n_{e}\left(\frac{3}{2} k_{B} T+m_{m} L_{v}+\frac{4}{3} \pi \sigma r_{e}{ }^{2}\right)
$$

which complete the mass and energy balances (equations (2.3) and (2.9)). It is assumed that the vapour molecule inside the bubble is monoatomic and $m_{m}$ is the atomic mass.

Figure 5 shows a schematic diagram of the temperature and pressure profiles in the liquid layer. From the actual pressure and saturation pressure profiles, the superheated zone and the subcooled zone can be calculated.

\subsection{Fluid flow}

Fluid flow, in general, is one of the most difficult parts in modelling laser-material processing. In the case of high density laser beams, it can be significantly simplified because of the thin liquid layer. It has been reported that the liquid layer thickness is of the order of several micrometres [13,14,31].

A simple scheme has been developed by integrating the Navier-Stokes equation over a computational cell (see figure 3 ) along the liquid layer as follows:

$$
\begin{gathered}
\left.\frac{1 \overline{\mathrm{d} v}}{\mathrm{~d} t}\right|_{i-1 / 2} ^{n+1}=\frac{1}{2} \rho_{i-1 / 2}\left[\overline{\left(v_{i-1}^{n}\right)^{2}}-\overline{\left(v_{i}^{n}\right)^{2}}\right]+\left(p_{i-1}^{n}-p_{i}^{n}\right) \\
+\frac{\Delta s_{i-1 / 2}^{n}}{h_{i-1 / 2}^{n}}\left(\left.\mu_{i-1 / 2} \frac{\partial u}{\partial n}\right|_{\phi, i-1 / 2} ^{n}-\left.\mu_{i-1 / 2} \frac{\partial u}{\partial n}\right|_{\psi, i-1 / 2} ^{n}\right)
\end{gathered}
$$

where

$$
\lambda=\frac{1}{\rho_{i-1 / 2} \Delta s_{i-1 / 2}^{n}} .
$$

Here the superscript $n$ and the subscript $i$ are used for time and space respectively, and the over-bars denote variables averaged across the liquid layer. The velocity gradient at the liquidvapour interface is as follows:

$$
\left.\mu_{i-1 / 2} \frac{\partial u}{\partial n}\right|_{\phi, i-1 / 2} ^{n}=\left.\left.\frac{\mathrm{d} \sigma}{\mathrm{d} T}\right|_{i-1 / 2} ^{n} \frac{\mathrm{d} T}{\mathrm{~d} s}\right|_{i-1 / 2} ^{n}
$$

where $\sigma$ denotes the surface tension. To complete the scheme, we have to obtain the velocity gradient at the solidliquid interface and the averaged squared velocity term in 


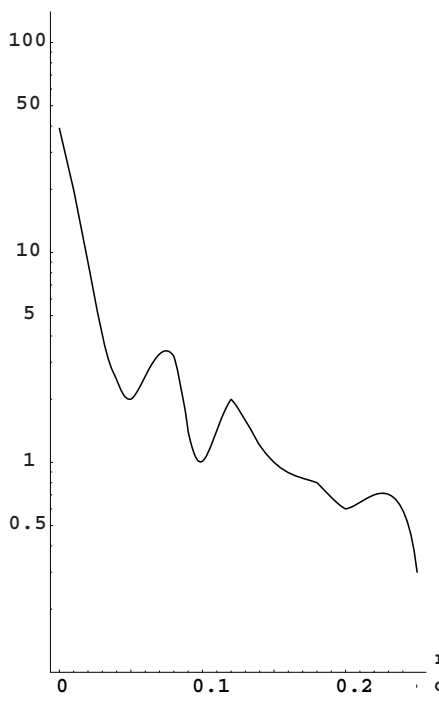

(A)

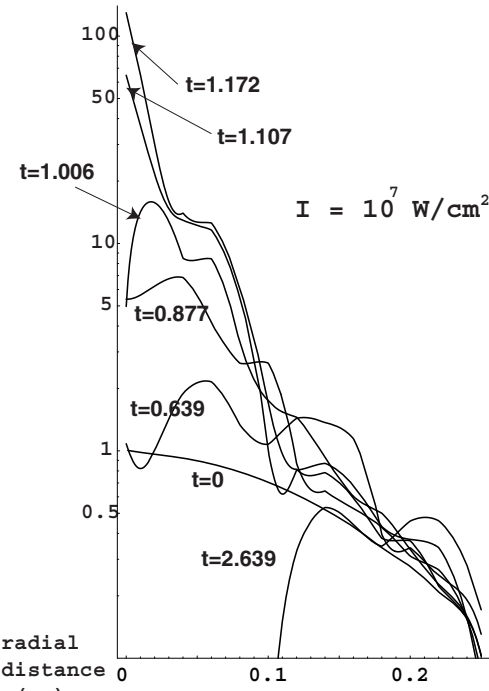

(B)

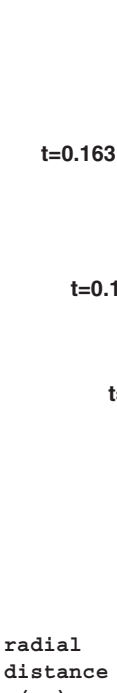

(mm)

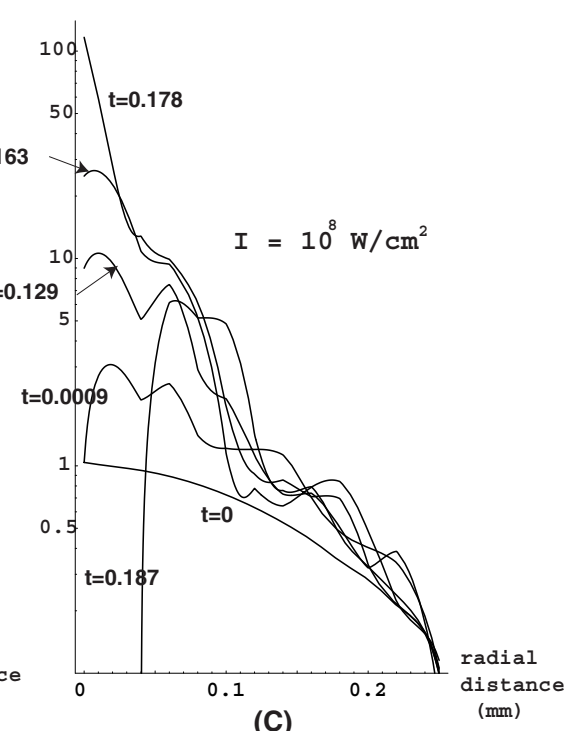

(C)

Figure 7. Effective laser intensity profile (A) for a paraboloid (depth $=1.5 \mathrm{~mm}$ ) and $(\mathrm{B}),(\mathrm{C})$ for self-evolving liquid-vapour interface profiles (the time, $t$, is in milliseconds).
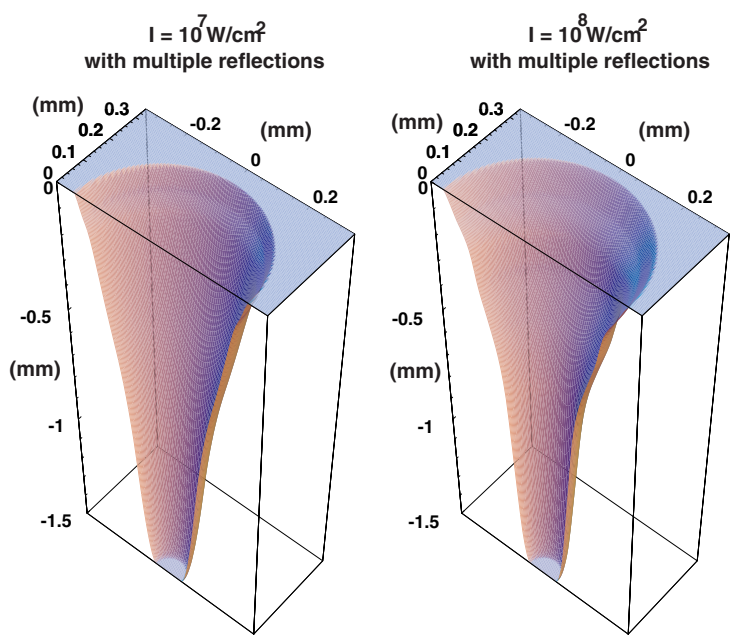

Figure 8. Three-dimensional hole profile with multiple reflections.

equation (2.18), and this can be achieved by assuming a velocity profile in the liquid layer. Since we know the velocity gradient at the liquid-vapour interface, the mean velocity $\overline{v_{i}^{n}}$ and the tangential velocity at the solid-liquid interface, which is zero, we can use the quadratic polynomial to interpolate these values. The velocity at the centre of the cell can be updated from the following relation:

$$
\overline{v_{i-1 / 2}^{n+1}}=\overline{v_{i-1 / 2}^{n}}+\left.\overline{\mathrm{d} v}\right|_{i-1 / 2} ^{n+1} \Delta t .
$$

Here $\Delta t$ is the time step.

\section{Results and discussion}

The model described above is implemented with a laser intensity of $10^{7}$ and $10^{8} \mathrm{~W} \mathrm{~cm}^{-2}$. A Gaussian spatial distribution is considered for $\mathrm{CW}$ laser. A ray tracing technique
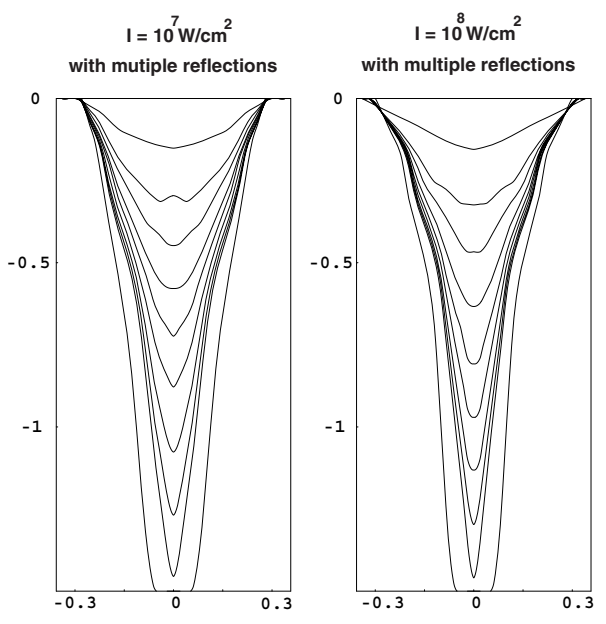

Figure 9. Transient laser-created hole profile with multiple reflections.

is used to model multiple reflections inside the vapour cavity, assuming that the diffuse reflection mode is negligible compared to the specular reflection. At both intensities, simulations are performed with and without the multiple reflection phenomena, in order to investigate its effects on the predictions. Plasma interaction and recondensation phenomenon are neglected here. An iron plate of $1.5 \mathrm{~mm}$ thickness is used as the substrate and properties of iron are given in table 1. Temperature-dependent material properties are used for simulation.

The effective laser absorptivity with time is presented in figure 6. It is clearly seen that the effective absorptivity increases as the liquid-vapour interface deepens. The energy utilization increases up to around $80 \%$ even though the initial absorptivity for a flat surface is very low. After penetration the effective laser absorptivity decreases as part of the laser energy is lost though the bottom hole. The time taken for complete penetration of a $1.5 \mathrm{~mm}$ thick plate with $10^{7}$ and 



Figure 10. Free surface temperature profile (time sequence: dark $\rightarrow$ light curves).

$10^{8} \mathrm{~W} \mathrm{~cm}^{-2}$ laser power densities were found to be 1.2 and $0.19 \mathrm{~ms}$, respectively. Experimental observations indicate that at a $10^{7} \mathrm{~W} \mathrm{~cm}^{-2}$ power density the penetration time is in fact in the order of a few milliseconds [32,33].

Figures 7(B) and (C) present the effective laser intensity profile variation with penetration depth. It is interesting to note that both the graphs for different laser intensities have similar shapes when they are normalized with the original intensity profile for a flat surface. The laser energy tend to concentrate near the centre, where the effective intensity reaches a value that is two orders of magnitude higher than the original distribution. It is well known that the redistribution of the laser energy inside the cavity occurs due to multiple internal reflections. However, the extent to which this redistribution occurs is not well understood. Most earlier studies [34] on multiple reflections used a paraboloid of revolution as the resulting shape of the cavity. In order to compare the effect of a dynamically evolving surface profile on the energy focusing characteristics, a paraboloid of revolution with the depth of $1.5 \mathrm{~mm}$ was used to simulate multiple reflections similar to earlier approaches.

As shown in figure 7(A), the maximum intensity occurring at the centre of the cavity is only around 34 times larger than the value for a flat surface [34]; but the consideration of the dynamically evolving surface in our simulation predicts a much higher concentration of energy at the centre. All the physics in laser-material processing, such as evaporation, fluid flow, heat transfer and solidification, are highly dependent on the energy deposition characteristics. Therefore, an a priori assumption of the paraboloid revolution for simulating multiple reflections within a laser-induced cavity would predict a completely different result from the present simulation. As will be demonstrated later, in the case of $10^{8} \mathrm{~W} \mathrm{~cm}^{-2}$ laser power density, homogeneous boiling can occur in the centre region according to the energy deposition pattern obtained from the present simulation; whereas the assumption of paraboloid revolution would not predict such phenomena. The influence

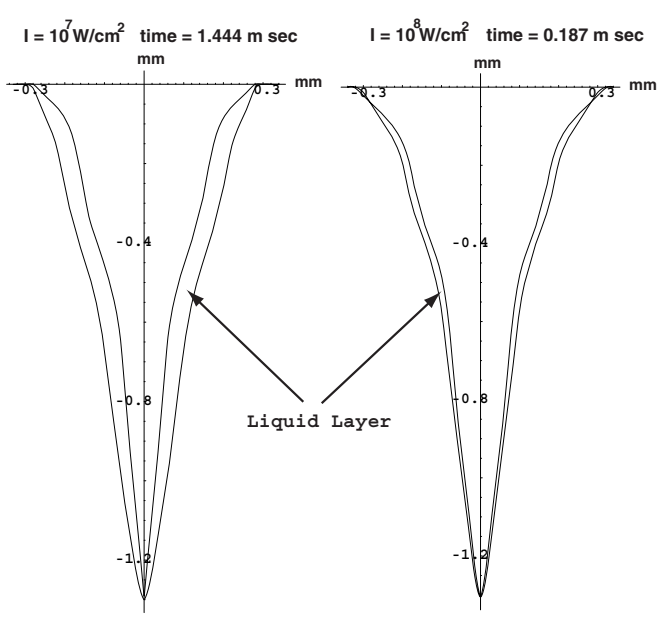

Figure 11. Liquid layer thickness against radial distance.

of the correct cavity geometry is demonstrated for the first time in the present work.

To demonstrate the capabilities and advantages of using the level-set method, fully penetrated three-dimensional hole profiles are presented in figure 8. The front evolution is a natural outcome of the scheme, even during full penetration. Unlike other tracking schemes, no reconstruction of the surface is needed here. Surface reconstruction, in particular, becomes difficult in three-dimensional simulations. So far, no other numerical studies have been able to predict a fully penetrated laser-induced cavity in three dimensions. In the present simulation, no numerical instability in particular was observed. We believe that the ability to implicitly couple the surface profile with the process physics simulation is a significant accomplishment.

Figure 9 shows the transient surface recession profile. It is observed that as the laser intensity increases, the cavity shape becomes narrower. Thus, to make very fine holes a 




Figure 12. Surface recession speed against radial distance.
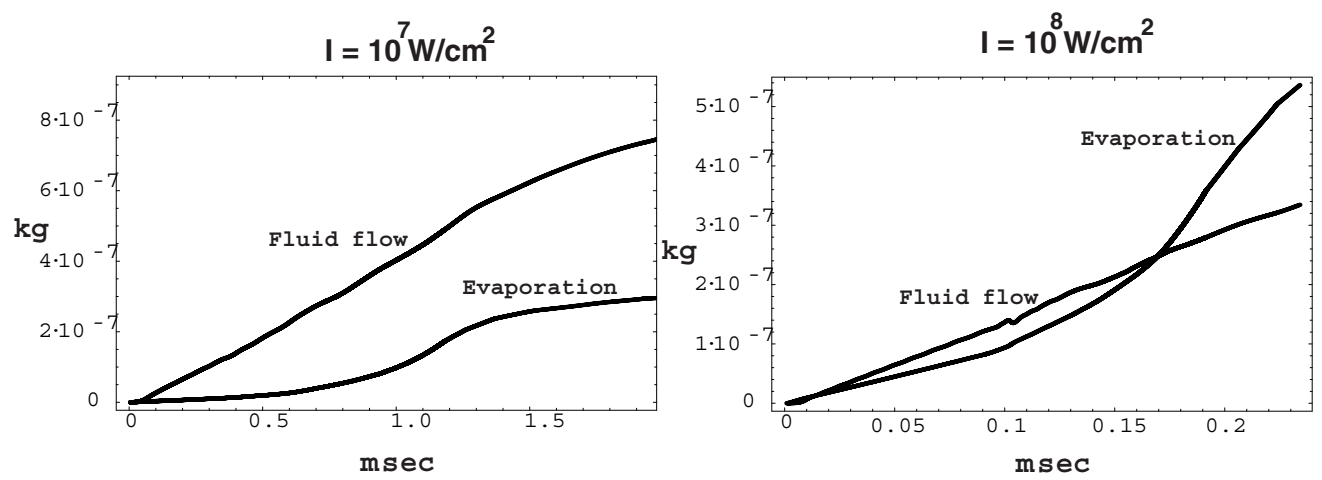

Figure 13. Accumulated mass loss ratio: flow and vaporization.

higher-intensity laser beam is required. This phenomenon has also been experimentally observed. As discussed above, multiple reflection phenomena have a marked effect at higher power densities in concentrating the beam energy near the centre. As will be seen below, the material loss mode also becomes more evaporative in nature at higher densities, leading to a smaller zone affected by heat or liquid layer thickness. Thus, the cavity becomes narrower and cleaner.

Figure 10 presents transient liquid-vapour surface temperature profiles. As seen from this figure, a steep temperature gradient exists at the central location and the temperature values are far beyond the normal boiling point. In the case of a $10^{8} \mathrm{~W} \mathrm{~cm}^{-2}$ laser beam, the maximum temperature is clearly in the regime of homogeneous boiling; but this phenomena is limited to the central zone. At about $0.8 T_{c}$ homogeneous nucleation begins due to the large amount of liquid superheat and a significant drop in the surface tension [1-5]. The electric conductivity of liquid metal drops slowly with temperature up to a certain point, and then sharply drops to virtually zero, transforming the liquid metal into a liquid dielectric [6-8]. This optically transparent zone propagates into the interior of the target like a wave [9]. Customarily, high-density laser beam-material interaction models have assumed the free surface temperature to be close to the equilibrium boiling temperature. To complete the Knudsen layer jump relations, a CC relation has been employed in almost all the research, including Knight's original work [26], assuming that a liquid-vapour interface is always saturated. However, our model predicts a much higher temperature, where the interface is highly superheated. There are experimental indications that free surface temperatures are in fact in a similar order as that predicted by our model; this is one of the most interesting predictions.
The effect of the laser power density on the liquid layer thickness is presented in figure 11. As the intensity increases, the liquid layer thickness decreases. Also from figure 11, it is observed that there is a monotonic increase in liquid layer thickness as the cavity depth deepens. The presence of a liquid layer around the laser-induced cavity is unwanted in many laser-aided material processing operations such as precision machining and drilling. Solidification of the liquid layer occurs at very rapid rate. As the thickness of the liquid increases, the chance of crack development also increases. Thus, to obtain a very thin recast layer, a high-intensity laser should be used. This observation has tremendous technological importance. Recently, significant advancement has been achieved in the area of high-peak-power, short-pulse laser development for precision machining that can avoid any significant recast layer formation.

Figure 12 shows surface recession speeds. As expected, the speed increases with laser intensity. A 10 times higher laser intensity increases the speed from four to 30 . This implies that surface recession speed might saturate at very high laser intensities due to surface temperatures reaching the near critical point. This saturation phenomena can be explained using the notion of induced transparency waves, but this is out of the scope of the present paper.

The cumulative mass loss rate due to fluid flow and evaporation are presented in figure 13 . With a $10^{7} \mathrm{~W} \mathrm{~cm}^{-2}$ laser intensity, fluid flow is dominant and the evaporation loss is negligible. On the other hand, with a $10^{8} \mathrm{~W} \mathrm{~cm}^{-2}$ laser intensity, the evaporative mass loss becomes greater than the fluid flow loss. In other words, with increasing power densities the process becomes evaporation dominated. This observation is in line with many experimental studies. 


\section{Conclusions}

The present simulation has demonstrated many interesting features of laser-material interaction phenomena. They can be summarized as follows.

(a) Effective laser absorptivity increases as the cavity deepens. In the present study the maximum energy absorbed by the cavity is found to be around $80 \%$ of the total input laser energy.

(b) Due to the multiple reflections the laser beam energy is highly concentrated at the centre of the cavity and the cavity shape is narrowed by approximately $50 \%$ compared to a cavity, with similar depth, formed without taking multiple reflections into account.

(c) The surface temperature is much higher than the normal boiling point, and with a laser intensity of $10^{8} \mathrm{~W} \mathrm{~cm}^{-2}$ the surface temperature approaches the homogeneous boiling regime with the help of multiple reflections.

(d) The liquid layer thickness increases as the hole deepens. The liquid layer thickness decreases by about $60 \%$ on increasing the laser intensity from $10^{7}$ to $10^{8} \mathrm{~W} \mathrm{~cm}^{-2}$.

(e) As the laser intensity increases, the mass loss mechanism changes from the fluid ejection mode to the evaporation dominant material removal mode.

(f) The level-set method successfully captures the free surface evolution even during full penetration, which is one of the major achievements.

\section{Acknowledgments}

This work was made possible by the continued support of the Office of Naval Research under grant no. N00014-97-1-0124. Dr George Yoder is the programme manager.

\section{References}

[1] Ross R and Greenwood D 1969 Prog. Mater. Sci. 14175

[2] Martynyuk M 1983 Russian J. Phys. Chem. 57494

[3] Kelly R and Miotello A 1996 Appl. Surf. Sci. 96-98 205

[4] Yilbas B S and Sami M 1997 J. Phys. D: Appl. Phys. 301996
[5] Song K H and Xu X 1998 Appl. Surf. Sci. 127-129 111

[6] Martynyuk M 1975 Russian J. Phys. Chem. 491545

[7] Hensel F 1990 J. Phys.: Condens. Matter 233

[8] Hensel F and Edwards P 1996 Physics World 943

[9] Batanov V, Bunkin F, Prokhorov A and Fedorov V 1973 Sov. Phys.-JETP 36311

[10] Peterlongo A, Miotello A and Kelly R 1994 Phys. Rev. E 50 4716

[11] Kelly R and Miotello A 1999 Phys. Rev. E 602616

[12] Craciun V, Craciun D, Boulmer-Leborgne C and Hermann J 1998 Phys. Rev. B 586787

[13] Chan C and Mazumder J 1987 J. Appl. Phys. 624579

[14] Kar A, Rockstroh T and Mazumder J 1992 J. Appl. Phys. 71 2560

[15] Modest M F 1996 Int. J. Heat Mass Transfer 39221

[16] Ganesh R, Faghri A and Hahn Y 1997 Int. J. Heat Mass Transfer $\mathbf{4 0} 3351$

[17] Ganesh R, Faghri A and Hahn Y 1997 Int. J. Heat Mass Transfer 403361

[18] Osher S and Sethian J A 1988 J. Comput. Phys. 7912

[19] Mulder W, Osher S and Sethian J A 1992 J. Comput. Phys. 100209

[20] Adalsteinsson D and Sethian J A 1995 J. Comput. Phys. 118 269

[21] Adalsteinsson D and Sethian J A 1995 J. Comput. Phys. 120 128

[22] Adalsteinsson D and Sethian J A 1995 J. Comput. Phys. 122 348

[23] Smiljanovski V, Moser V and Klein R 1997 Combust. Theory Modelling 1183

[24] Sethian J A 1996 Level Set Methods (Cambridge: Cambridge University Press)

[25] Carslaw H and Jaeger J 1959 Conduction of Heat in Solids 2nd edn (Oxford: Oxford University Press)

[26] Knight C J 1979 AIAA J. 17519

[27] Ytrehus T and Ostmo S 1996 Int. J. Multiphase Flow 22 133

[28] Carey V P 1992 Liquid-Vapor Phase Change Phenomena (Bristol, PA: Hemisphere)

[29] Skripov V 1974 Metastable Liquids (New York: Wiley)

[30] Debenedetti P G 1996 Metastable Liquids Concepts and Principles (Princeton, NJ: Princeton University Press)

[31] Kar A and Mazumder J 1990 J. Appl. Phys. 683884

[32] Duffey T, Choi J and Mazumder J 1999 Welding Res. at press

[33] Duley W Laser Welding (New York: Wiley)

[34] Wei P and Ho C 1998 Int. J. Heat Mass Transfer 413299

[35] Beutl M, Pottlacher G and Jäger H 1994 Int. J. Thermophys. 151323 